\title{
Finite element modelling of the interphase region on the mechanical behaviour of a composite containing micrometer sized spherical particles
}

\author{
K. N. $\operatorname{Tran}^{1} \quad$ Y. Ding ${ }^{2} \quad$ J. A. Gear ${ }^{3}$
}

(Received 16 October 2009; revised 22 February 2010)

\begin{abstract}
When a particle or filler is embedded into a polymer matrix, a third region is generated between the filler and matrix due to their chemical reaction between. This region is known as the interphase region. The properties of the interphase are affected by the material properties of the particles and the polymer matrix as well as the volume fraction of the composite. We implement the interphase mathematical model into the finite element method algorithm to investigate the effect of the interphase region on the deformation behaviour of the composite. The properties of the interphase at the matrix boundary are assumed to be smooth and continuous, while discontinuous at the particle boundary. The finite element algorithm employs Galerkin weak formulation with quadratic shape functions. The displacement, strain and stress of the composite are calculated along the radial axis originated from the
\end{abstract}

http://anziamj . austms.org.au/ojs/index.php/ANZIAMJ/article/view/2526 gives this article, (C) Austral. Mathematical Soc. 2010. Published February 25, 2010. ISSN 1446-8735. (Print two pages per sheet of paper.) 
centre of a particle. The finite element modelling results are presented.

\section{Contents}

1 Introduction

2 The interphase model

C18

3 Formulation of displacement field

C20

4 The finite element algorithm

5 Result and discussion

5.1 Material properties ................ C23

5.2 Results and discussions . . . . . . . . . . . . .

6 Conclusion

C29

References

C29

\section{Introduction}

Adding fillers to a polymer matrix was only meant to decrease the price of the product. However, soon it was discovered that the properties of the resultant composite altered significantly from the properties of the matrix polymer, such as flame retardancy [5], improved overall composite properties, decreased specific heat, increased heat conductivity, increased stiffness properties and strength $[1,5]$.

The overall properties of a particle-reinforced composite are difficult to determine due to the complex bonding between the filler and matrix. In some 
research a nonrealistic perfect bonding between the filler and matrix was assumed $[6,9]$. A third region, the interphase, is generated when fillers are added to a polymer matrix. The effects of the interphase on the overall properties of the composite include changing the elastic properties $[1,3]$, thermal expansion coefficient [4] and stress and strain behaviors [8]. In the authors' previous work $[1,7]$, an interphase mathematical model was established based on a one dimensional, three phase, composite model. The mathematical model describes the properties and behaviour of the inhomogeneous interphase region between a spherical micrometer sized particle and a polymer matrix. Using the interphase mathematical model, the shear modulus and Young's modulus were calculated and compared well with experimental results.

The one dimensional interphase mathematical model is implemented into the Finite Element Method (FEM) algorithm based on the Galerkin Weak formulation with quadratic shape functions. The displacement, strain and stress distributions in the composite were obtained and presented.

\section{The interphase model}

The model of a representative portion of a three phase composite is shown in Figure 1(a). When a spherical filler of radius $r_{f}$ is added to a polymer matrix, an annular interphase region with the inner radius $r_{f}$ and outer radius $r_{i}$ is created between the filler and the polymer matrix, of which the outer radius is $r_{m}$ (Figure 1(a)). The material properties of the filler and polymer matrix, such as the Young's Modulus, Poison ratios and coefficients of thermal expansion, are isotropic and of different constant values. However, the properties of the interphase region are inhomogeneous [2,4] and some functions of the spatial variable $r\left(r_{f} \leqslant r \leqslant r_{i}\right)$. The rate of change of an interphase property at the boundary of the filler side is non-smooth and discontinuous; while at the matrix side is smooth and continuous. Letting $F_{\text {int }}(r)$ represent a property of the interphase region, the smooth nature of $F_{\text {int }}(r)$ at the bound- 


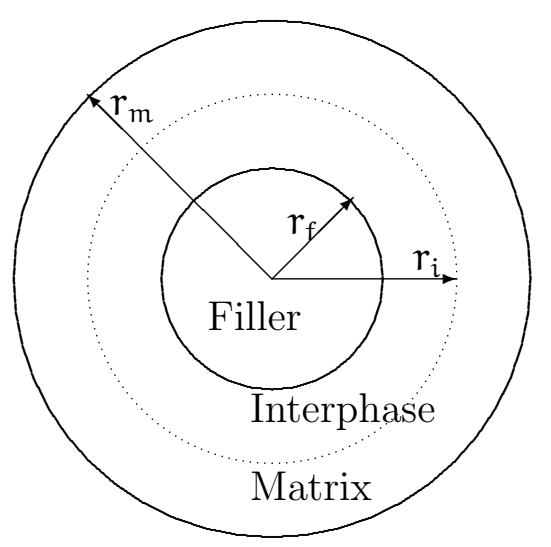

(a) The model of three phase composite

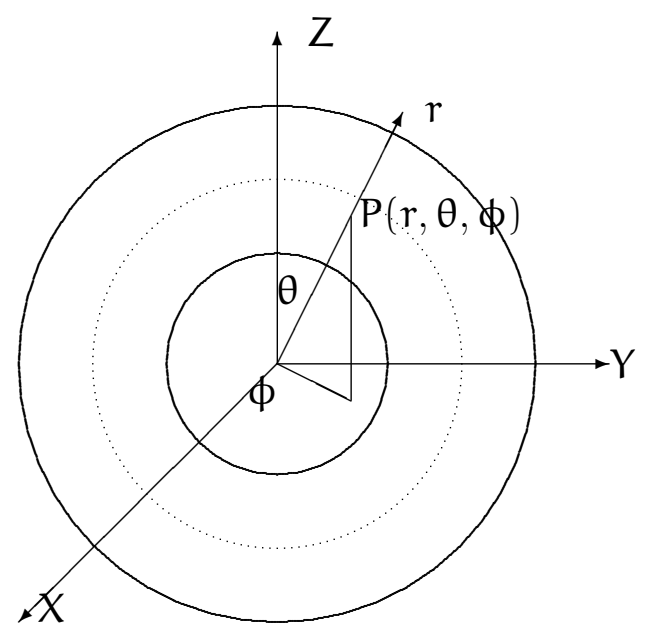

(b) three phase composite in a spherical coordinates $(r, \theta, \phi)$

Figure 1: Three phase spherical filler composite.

ary of the polymer matrix side gives $d F_{\text {int }}\left(r_{i}\right) / d r=0$. Thus, we defined the governing equation of the interphase for the property [1]

$$
\frac{d F_{i n t}(r)}{d r}=A r^{\eta}\left(r_{i}-r\right) e^{\gamma r}, \quad r \in\left[r_{f}, r_{i}\right],
$$

where parameters $\eta$ and $\gamma$ are arbitrary integers, and for simplicity, $\eta=1$ and $\gamma=1$ were chosen [1]. The boundary conditions for equation (1) are

$$
\mathrm{F}_{\text {int }}\left(\mathrm{r}_{\mathrm{f}}\right)=\mathrm{kF}_{\mathrm{f}}, \quad \mathrm{F}_{\mathrm{int}}\left(\mathrm{r}_{\mathrm{i}}\right)=\mathrm{F}_{\mathrm{m}},
$$

where $F_{f}$ is a property of the filler; $F_{m}$ is a property of the polymer matrix; and $\mathrm{k}$ is a positive constant. A property of the composite is discontinuous at the boundary of the filler and interphase except for $k=1$, for which it is continuous as $F_{\text {int }}(r)=F_{f}$. Using the ratio $J=F_{\text {int }}\left(r_{f}\right) / F_{m}$ to describe the state of discontinuity at the boundary of the filler and interphase [4], for a given $J$, the value of $k=J \cdot F_{m} / F_{f}$. The arbitrary constant $A$ in equation (1) is determined once the state of discontinuity at the boundary of the filler and interphase is defined. 
Solving equation (1) subject to the boundary conditions (2), the following solution is obtained

$$
F_{\text {int }}(r)=A\left\{e^{r}\left[-r^{2}+\left(r_{i}+2\right)(r-1)\right]+e^{r_{i}}\left(2-r_{i}\right)\right\}+F_{m}, \quad r \in\left[r_{f}, r_{m}\right],
$$

where

$$
A=\frac{k F_{f}-F_{m}}{e^{r_{f}}\left[-r_{f}^{2}+\left(r_{i}+2\right)\left(r_{f}-1\right)\right]+e^{r_{i}}\left(2-r_{i}\right)} .
$$

Equation (3) describes a property of the interphase region as a function of the spatial variable $r$.

\section{Formulation of displacement field}

The three phase composite model is analysed in the spherical polar coordinates $(r, \theta, \phi)$ shown in Figure 1(b). Suppose that, by some means, the composite is deformed so that its points undergo displacement. Let the displacement of an arbitrary point be $\overrightarrow{\mathfrak{u}}=\left(\mathfrak{u}_{r}, \mathfrak{u}_{\theta}, \mathfrak{u}_{\phi}\right)$ referred to axes $r, \theta$ and $\phi$, respectively. When the temperature is constant and deformation is spherically symmetric, the displacement of the composite is independent of $\theta$ and $\phi$. The strain $\varepsilon$ is

$$
\varepsilon_{r}=\frac{d u_{r}}{d r}, \quad \varepsilon_{\theta}=\varepsilon_{\phi}=\frac{u_{r}}{r}, \quad \varepsilon_{\theta \phi}=\varepsilon_{\phi r}=\varepsilon_{r \theta}=0,
$$

where $\varepsilon_{r}, \varepsilon_{\theta}$ and $\varepsilon_{\phi}$ are the normal strains and $\varepsilon_{\theta \phi}, \varepsilon_{\phi r}$ and $\varepsilon_{r \theta}$ are the shear strains. Consequently, the stress is determined from Hooke's law:

$$
\begin{aligned}
& \sigma_{\theta \phi}=\sigma_{r \phi}=\sigma_{r \theta}=0, \\
& \sigma_{r}=\frac{E}{(1+v)(1-2 v)}\left[(1-v) \varepsilon_{r}+v \varepsilon_{\theta}+v \varepsilon_{\phi}\right], \\
& \sigma_{\theta}=\sigma_{\phi}=\frac{E}{(1+v)(1-2 v)}\left[\varepsilon_{\theta}+v \varepsilon_{r}\right],
\end{aligned}
$$


where $\sigma_{\mathrm{r}}, \sigma_{\theta}$ and $\sigma_{\phi}$ are the axial stresses, while $\sigma_{\theta \phi}, \sigma_{\phi \mathrm{r}}$ and $\sigma_{\mathrm{r} \theta}$ are the shear stresses. E and $v$ are the Young's modulus and Poison ratio, respectively.

Substituting equation (4) into equation (5) gives the stress in terms of displacement in the radial direction only

$$
\sigma_{r}=X \frac{d u_{r}}{d r}+Y \frac{u_{r}}{r}, \quad \sigma_{\theta}=Z \frac{d u_{r}}{d r}+W \frac{u_{r}}{r},
$$

where

$$
\begin{aligned}
& X=X_{\omega}=\frac{\left(1-v_{\omega}\right) E_{\omega}}{\left(1+v_{\omega}\right)\left(1-2 v_{\omega}\right)}, \quad Y=Y_{\omega}=\frac{2 v_{\omega} E_{\omega}}{\left(1+v_{\omega}\right)\left(1-2 v_{\omega}\right)}, \\
& W=W_{\omega}=\frac{X_{\omega}}{1-v_{\omega}}, \quad Z=Z_{\omega}=\frac{Y_{\omega}}{2} \quad \text { for } \omega=f, i, m .
\end{aligned}
$$

Note that the material properties of the filler and polymer matrix $E_{f}, E_{m}, \mu_{f}$ and $\mu_{m}$ are known constants, while the Young's modulus and Poison ratios of the interphase $E_{i}$ and $\mu_{i}$ are the variables of $r$ and determined by equation (3). The equilibrium equation for the stress distribution of the composite is

$$
\begin{aligned}
& \frac{d}{d r}\left(X \frac{d u_{r}}{d r}\right)+\frac{d}{d r}\left(Y \frac{u_{r}}{r}\right)+\frac{2(X-Z)}{r} \frac{d u_{r}}{d r}+\frac{2(Y-W)}{r^{2}} u_{r}=0, \\
& u_{r}(0)=0, \quad u_{r}\left(r_{m}\right)=\bar{\varepsilon} r_{m} .
\end{aligned}
$$

\section{The finite element algorithm}

The interphase mathematical model is implemented into the FEM algorithm to calculate the displacement along the radial axis ( $\mathrm{r}$ direction). We discretize the $r$ domain into $N$ quadratic elements with a total nodal number of $2 \mathrm{~N}+1$, Taking the piecewise quadratic functions $\psi_{p}(r)$ as the weighting functions and $r_{p}$ the nodal coordinate of the corresponding node $p$, the weak formulation of equation (6) is 


$$
\begin{aligned}
\int_{r_{1}}^{r_{2 N+1}}\left[\frac{d}{d r}\left(X \frac{d u_{r}}{d r}\right)+\frac{d}{d r}\left(Y \frac{u_{r}}{r}\right)+\right. & \frac{2(X-Z)}{r} \frac{d u_{r}}{d r} \\
& \left.+\frac{2(Y-W)}{r^{2}} u_{r}\right] \psi_{p}(r) d r=0,
\end{aligned}
$$

for $p=1,2, \ldots, 2 \mathrm{~N}+1$. Considering the essential boundary conditions at both ends, we simplify equation (7) by integrating by parts to obtain

$$
\begin{aligned}
\int_{r_{1}}^{r_{2 N+1}}\left[\left(\frac{2(X-Z)}{r} \frac{d u_{r}}{d r}+\frac{2(Y-W)}{r^{2}} u_{r}\right) \psi_{p}(r)\right. & \\
& \left.-\left(X \frac{d u_{r}}{d r}+Y \frac{u_{r}}{r}\right) \frac{d \psi_{p}(r)}{d r}\right] d r=0 .
\end{aligned}
$$

The approximate solution is assumed to be $u_{r}=\sum_{q=1}^{2 N+1} u_{q} \psi_{q}(r)$ where $U_{q}$ $(q=1,2, \ldots, 2 N+1)$ are the nodal displacements. Substituting into equation (8) yields the following set of discretised equations

$$
\mathrm{M}_{\mathrm{p}, \mathrm{q}} \mathrm{U}_{\mathrm{q}}=\mathrm{L}_{\mathrm{p}} \quad \text { for } \mathrm{p}, \mathrm{q}=1,2, \ldots, 2 \mathrm{~N}+1,
$$

where

$$
\begin{aligned}
M_{p, q}=\int_{r_{1}}^{r_{2 N+1}\left[\left(\frac{2(X-Z)}{r} \frac{d \psi_{q}(r)}{d r}\right.\right.} & \left.+\frac{2(Y-W)}{r^{2}} \psi_{q}(r)\right) \psi_{p}(r) \\
& \left.-\left(X \frac{d \psi_{q}(r)}{d r}+\frac{Y}{r} \psi_{q}(r)\right) \frac{d \psi_{p}(r)}{d r}\right] d r
\end{aligned}
$$

and

$$
L_{p}= \begin{cases}0, & \text { if } p \neq 2 N+1, \\ \bar{\varepsilon} r_{m}, & \text { if } p=2 N+1\end{cases}
$$

Note that the regions of the filler, interphase and matrix are discretised into $n, m$ and $l$ elements with the equal length $h_{1}=r_{f} / n, h_{2}=\left(r_{i}-r_{f}\right) / m$ and $h_{3}=\left(r_{m}-r_{i}\right) / l$ in each region, respectively.

The axial strain and axial stress are found using $\varepsilon_{r}=\partial u_{r} / \partial r$ and $\sigma=E \mathcal{E}$, once equation (9) is solved for the displacement. 
TABle 1: Young's modulus and Poison ratio for E-glass and BISGMa/ TEGDMA epoxy

\begin{tabular}{lcc}
\hline Property & E-glass filler & BISGMA/TEGDMA epoxy \\
\hline Young's modulus E $(\mathrm{GPa})$ & 75 & 1.7 \\
Poison ratio $v$ & 0.24 & 0.35 \\
\hline
\end{tabular}

\section{$5 \quad$ Result and discussion}

\subsection{Material properties}

The biocompatible composite, E-glass particle reinforced BISGMA/TEGDMA epoxy, is used in the present study $[1,6]$. The average diameter of the Eglass particles is $2.6 \mu \mathrm{m}$. The Young's moduli and Poison ratios of the E glass particles and the BISGMa/TEGDMA polymers are listed in Table 1. According to these properties, the average thickness of the interphase is $2.4 \%$ of the radius of the filler $[1,6]$. For an iso-displacement loading, a small strain of $\bar{\varepsilon}=0.1 \%$ is used [6].

\subsection{Results and discussions}

Figures 2(a) and 2(b) show the variations of Young's modulus and Poison ratio over the interphase region for different $J$ values. The volume fraction of the filler is assumed as 20\%. When $J>1$, the Young's modulus of the interphase decreases moving away from the particle, while the Poison ratio increases. When $J<1$, the Young's modulus only increases slightly but the Poison ratio increases rapidly. In both cases, the Young's modulus and Poison ratio are continuous and smoothly merge with the polymer matrix.

Figures $3(\mathrm{a})$ and $3(\mathrm{~b})$ show the normalized radial displacement $\boldsymbol{u}_{\mathbf{r}} / \mathbf{u}\left(\boldsymbol{r}_{\mathbf{m}}\right)$ versus the normalized radial distance $r / r_{m}$ from the centre of the filler to 


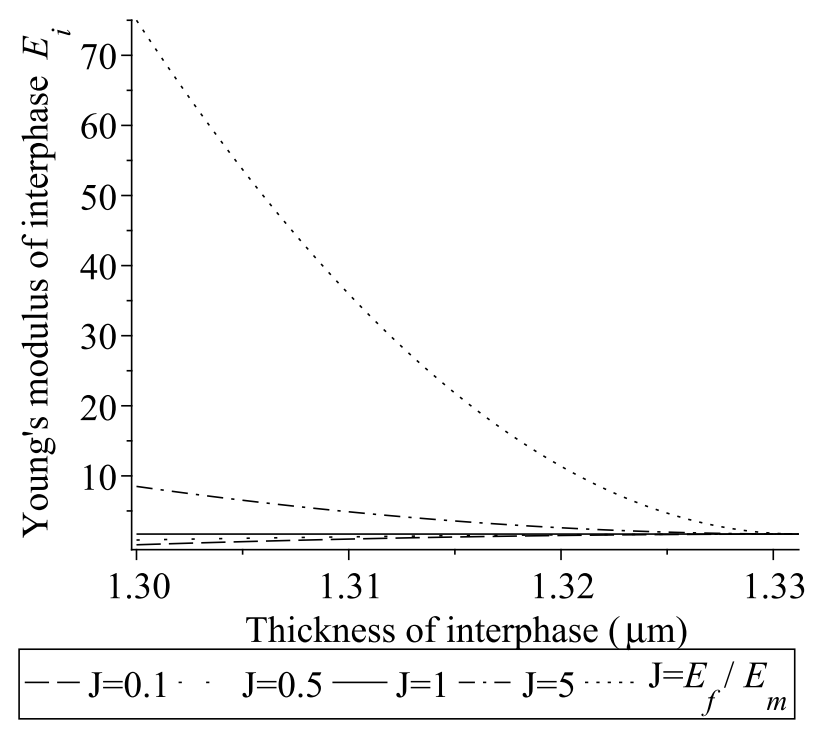

(a) Young's modulus

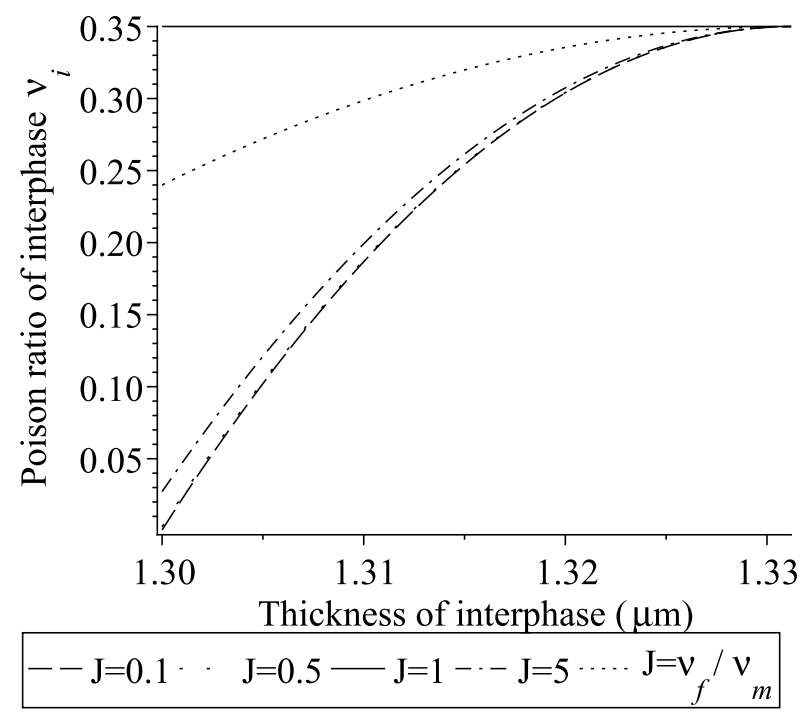

(b) Poison ratio

Figure 2: Variations of Young's modulus (a) and Poison ratio (b) over the interphase region. Interphase thickness $2.4 \%$ 


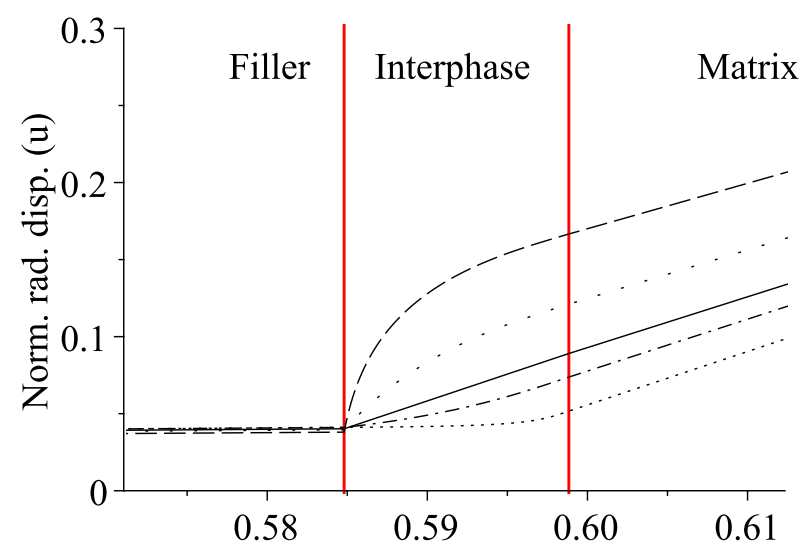

Norm. rad. dist. from filler centre

$--\mathrm{J}=0.1 \cdots \mathrm{J}=0.5-\mathrm{J}=1-\cdot \mathrm{J}=5 \cdots \cdot \mathrm{J}=F_{f} / F_{m}$

(a) Volume fraction of fillers: $20 \%$

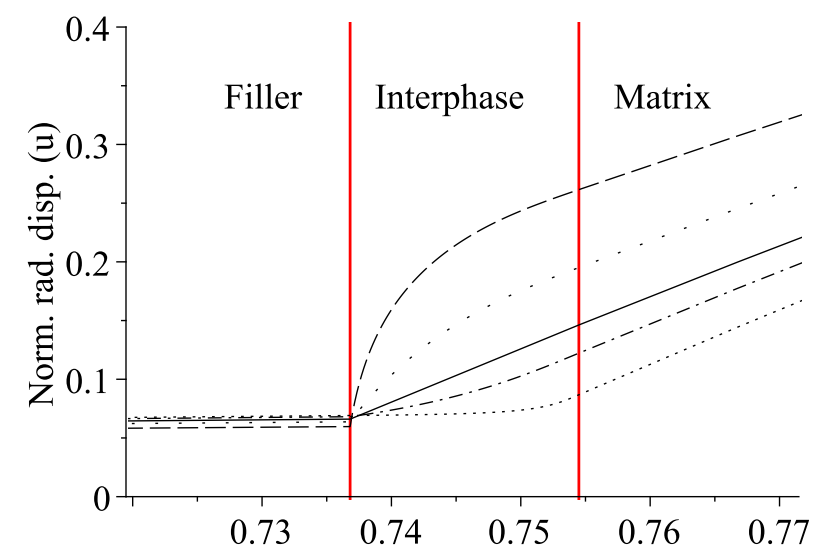

Norm. rad. dist. from filler centre

$--\mathrm{J}=0.1 \cdots \mathrm{J}=0.5-\mathrm{J}=1-\cdots \mathrm{J}=5 \cdots{ }_{f} \quad \mathrm{~J}=F_{f} / F_{m}$

(b) Volume fraction of fillers: $40 \%$

FIgURE 3: Normalised radial displacement against radial distance. 
the outer surface of the matrix for the volume fractions $20 \%$ and $40 \%$, respectively. Figure 3(a) shows that the displacement is independent of $r$ in the filler region, though an increase of the J value results in a slight decrease of the displacement. In the interphase region, the displacement becomes a function of $r$ and it increases more significantly for the cases of $J<1$. The displacement is continuous at both boundaries of the filler-interphase and interphase-matrix regardless of the $\mathrm{J}$ value. However, the displacement at the boundary of the filler and interphase is non-smooth, while smooth at the interphase-matrix boundary. Only for the case of $J=F_{f} / F_{m}$, the displacement is continuous and smooth at both boundaries of the filler-interphase and interphase-matrix. Figures $3(\mathrm{a})$ and $3(\mathrm{~b})$ also show that the volume fraction of fillers of a composite has a great influence on the displacement in the composite. The larger the volume fraction of fillers is, the greater increase in the magnitude of displacement.

Figures 4(a) and 4(b) show the strain variations in the composite for volume fractions of $20 \%$ and $40 \%$. The strain is normalized to the strain at the outer surface of the matrix $\varepsilon / \varepsilon_{\mathfrak{m}}$. The strain in the filler region is small for all $\mathrm{J}$. However, the strain distribution in the interphase region is strongly influenced by the value of $J$. When $J>1$, the strain decreases rapidly as $r$ increases, while the strain increases slowly with $J<1$. The strain at the filler-interphase boundary is discontinuous for all values of $J$ except the case of $J=F_{f} / F_{m}$, for which the strain seems continuous and smooth. The graphs also show the strain is least in the filler region. The results of the stress distribution in a composite are presented in Figures 5(a) and 5(b). As shown in Figure 5(a), the stresses in the filler region and matrix region are constants of different values, higher magnitudes in the filler region. At the boundary of filler and interphase, stresses are discontinuous for all the values of J except the case of $J=F_{f} / F_{m}$. The magnitude of the stress decreases as $r$ increases towards the boundary of the interphase and matrix. Figure 5(b) presents the stress distribution when the volume fraction is increased to $40 \%$. Overall, the stress behaves similar to the case in Figure 5(a) but the magnitudes of the stress are decreased. 


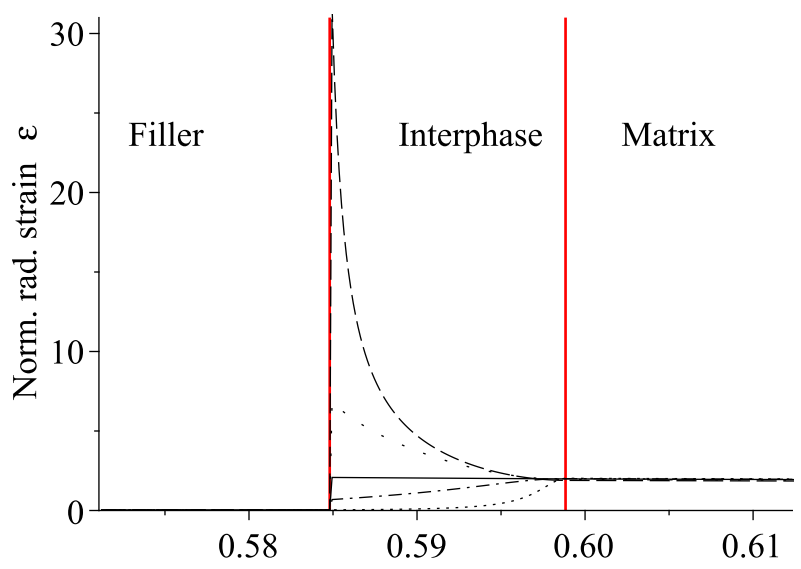

Norm. rad. dist. from filler centre

$$
--\mathrm{J}=0.1 \cdots \quad \mathrm{J}=0.5-\mathrm{J}=1-\cdot \mathrm{J}=5 \cdots . \mathrm{J}=F_{f} / F_{m}
$$

(a) Volume fraction of fillers $20 \%$

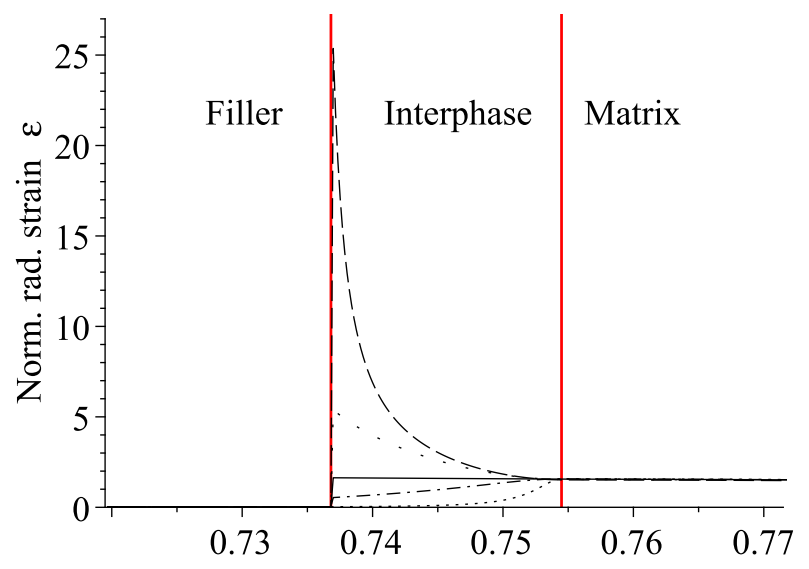

Norm. rad. dist. from filler centre

$$
--\mathrm{J}=0.1 \cdots \quad \mathrm{J}=0.5-\mathrm{J}=1-\cdot \mathrm{J}=5 \cdots \cdots \mathrm{J}=F_{f} / F_{m}
$$

(b) Volume fraction of fillers $40 \%$

FIgURE 4: Normalised radial strain against radial distance. 


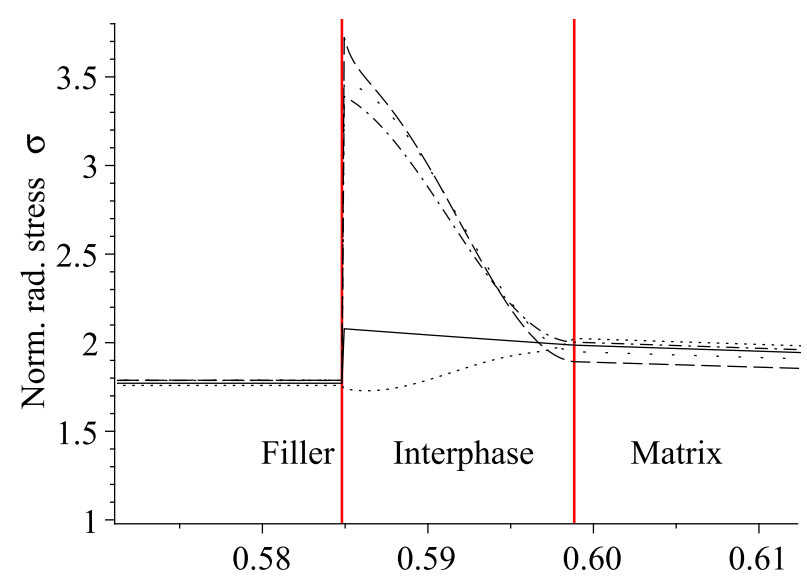

Norm. rad. dist. from filler centre

$--\mathrm{J}=0.1 \cdots \mathrm{J}=0.5-\mathrm{J}=1-\cdot \mathrm{J}=5 \cdots \mathrm{J}=F_{f} / F_{m}$

(a) Volume filler fraction $20 \%$

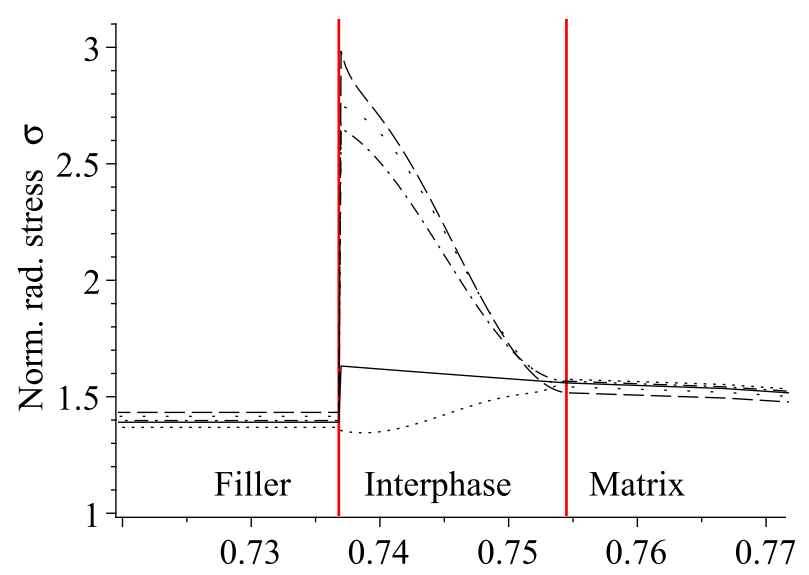

Norm. rad. dist. from filler centre

$--\mathrm{J}=0.1 \cdots \mathrm{J}=0.5-\mathrm{J}=1-\cdot \mathrm{J}=5 \cdots \mathrm{J}=F_{f} / F_{m}$

(b) Volume filler fraction $40 \%$

FiguRE 5: Normalized radial stress against radial distance. 


\section{Conclusion}

The new mathematical model describing a property of the interphase between a spherical particle and polymer matrix provides a good solution for predicting the mechanical properties of a composite.

The Young's modulus and Poison ratio vary over the interphase region as the state of discontinuity varies, measured by the J values. Overall, the Young's modulus of the composite decreases as the J value increases, while Poison ratio increases.

The FEM solution of the mathematical model provides a good prediction of the variation of displacement, strain and stress along the radial axis over the interphase region at different states of discontinuity.

The increase of the volume fraction of the filler results in decrease of the strain and stress, and increase of the displacement in the composite.

\section{References}

[1] Y. Ding, K. N. Tran, J. A. Gear, D. Mainwaring, and P. Murugaraj. Influence of the inetrphase between nanoparticle and matrix on Young's Mdulus of nanocomposite. Proceedings of the 2008 International Conference on Nanoscience and Nanotechnology, ICONN 2008, IEEE Cat. No. 08EX1890C, ISBN 1-4244-1504-7, Copyright 2008 IEEE, 28-31. http:

//ieeexplore. ieee.org/stamp/stamp. jsp?arnumber=04639213 C17, C18, C19, C23

[2] Z. Hashin and S. Shtrikman. A variational approach to the theory of the Elastic behaviour of mutiphase materials. Journal of the Mechanics and Physics of Solids 11, 1963, 127-140. doi:10.1016/0022-5096(63)90060-7 C18 
[3] Z. Hashin and P. J. M Moteiro. An inverse method to determine the elastic properties of the interphase between the aggregate and the cement paste. Cement and Concerte Research 32, 2002, 1291-1300. doi:10.1016/S0008-8846(02)00792-5 C18

[4] N. Lombardo Effect of an inhomogenoueus interphase on the thermal expansion coefficient of a particulate composite. Composite Science and Technology 65, 2005, 909-919. doi:10.1016/j.compscitech.2005.05.006 C18, C19

[5] J. Moczo and B. Pukanszky Polymer micro and nanocomposite: Structure, interactions, properties. Journal of Industrial and Engineering Chemistry 14, 2008, 535-563. doi:10.1016/j.jiec.2008.06.011 C17

[6] C. Sun, P. Saffarri, E. Ranade, K. Sadeghipour and G. Baran Finite Element analysis of elastic property bounds of a composite with randomly distributed particles. Composite Part A: Applied science and manufacturing 38, 2007, 80-86. doi:10.1016/j.compositesa.2006.01.010 C18, C23

[7] K. N. Tran and Y. Ding The effect of interphase on the properties of a composite containing spherical particles. Mathematics and Statistics Research Report No. 2007/02, School of Mathematical and Geospatial Sciences, RMIT University, 2007, ISBN: 978192116670 9. C18

[8] C. P. Tsui, C. Y. Tang and T. C. Le Finite element analysis of polymer composite filled by interphase coated particles. J. Material processing Technology 117, 2001, 105-110. doi:10.1016/S0924-0136(01)01117-7 C18

[9] C. Yang, H. Huh and T. H. Hahn. Evaluation of effective material peoperties of composite materials using FEM. J. Material processing Technology 140, 2003, 185-190. doi:10.1016/S0924-0136(03)00712-X C18 


\section{Author addresses}

1. K. N. Tran, School of Mathematical and Geospatial Sciences, RMIT University, Melbourne, Australia.

mailto:tran.kimngoc.k@edumail.vic.gov.au

2. Y. Ding, School of Mathematical and Geospatial Sciences, RMIT University, Melbourne, Australia.

mailto:yan.ding@rmit.edu.au

3. J. A. Gear, School of Mathematical and Geospatial Sciences, RMIT University, Melbourne, Australia. 